\title{
Immobilization of Neocallimastix patriciarum xylanase on artificial oil bodies and statistical optimization of enzyme activity
}

\author{
Ying-Jing Hung ${ }^{a}$, Chi-Chung Peng ${ }^{\mathrm{b}, 1}$, Jason T.C. Tzen ${ }^{\mathrm{c}}$, Ming-Ju Chen ${ }^{\mathrm{a}, *}$, Je-Ruei Liu ${ }^{\mathrm{a}, \mathrm{d}, *}$ \\ a Department of Animal Science and Technology, National Taiwan University, No. 50, Lane 155, Sec. 3, Keelung Rd., Taipei 106, Taiwan \\ ${ }^{\mathrm{b}}$ Department of Biotechnology, National Formosa University, Taiwan \\ ${ }^{\mathrm{c}}$ Graduate Institute of Biotechnology, National Chung-Hsing University, Taiwan \\ ${ }^{\mathrm{d}}$ Institute of Biotechnology, National Taiwan University, Taiwan
}

\section{A R T I C L E I N F O}

\section{Article history:}

Received 19 February 2008

Received in revised form 3 April 2008

Accepted 6 April 2008

Available online 20 May 2008

\section{Keywords:}

Immobilization

Neocallimastix patriciarum

Xylanase

Artificial oil body

\begin{abstract}
A B S T R A C T
A thermally stable and alkalophilic xylanase, XynCDBFV, from Neocallimastix patriciarum was overexpressed in Escherichia coli as a recombinant protein fused to the N-terminus of oleosin, a unique structural protein of seed oil bodies. As a result of the reconstitution of the artificial oil bodies (AOBs), the immobilization of active xylanase was accomplished. Response surface methodology (RSM) was employed for the optimization of the immobilized xylanase activity. The central composite design (CCD) and regression analysis methods were effective for determination of optimized temperature and $\mathrm{pH}$ conditions for the AOB-immobilized XynCDBFV. The optimal condition for the highest immobilized xylanase activity (3.93 IU/mg of total protein) was observed at $59^{\circ} \mathrm{C}$ and $\mathrm{pH}$ 6.0. Further, AOB-immobilized XynCDBFV retained $50 \%$ of its maximal activity after $120 \mathrm{~min}$ at $60{ }^{\circ} \mathrm{C}$, and it could be easily and simply recovered from the surface of the solution by brief centrifugation, and could be reused eight times while retaining more than $60 \%$ of its activity. These results proved it is a simple and effective method for direct immobilization of xylanases.
\end{abstract}

(c) 2008 Elsevier Ltd. All rights reserved.

\section{Introduction}

Xylan, a heteropolysaccharide containing substituent groups of acetyl, 4-O-methyl-D-glucuronosyl and $\alpha$-arabinofuranosyl residues linked to a backbone of $\beta-1,4$,-linked xylopyranose units, is the second-most-abundant renewable resource, with a high potential for degradation to useful end products (Subramaniyan and Prema, 2002). As xylan varies in structure between different plant species, complete hydrolysis requires a large variety of cooperatively acting enzymes such as xylanases, xylosidases, arabinofuranosidases, glucuronidases, acetylxylan esterases, ferulic acid esterases, and p-coumaric acid esterases (Kulkarni et al., 1999; Subramaniyan and Prema, 2002; Collins et al., 2005). All of these enzymes act cooperatively to convert xylan into its constituent sugars. Of these, xylanase is of particular significance because it can catalyze the random endohydrolysis of $\beta-1,4$-xylosidic linkages in xylan to produce xylooligosaccharides and xylose (Wu et al., 2006).

\footnotetext{
* Corresponding authors. Address: Institute of Biotechnology, National Taiwan University, 4 F., No. 81, Chang-xing Street, Taipei 106, Taiwan. Tel.: +886 2 33666011; fax: +886233666001 (J.-R. Liu).

E-mail addresses: cmj@ntu.edu.tw (M.-J. Chen), jrliu@ntu.edu.tw (J.-R. Liu).

${ }^{1}$ Equal contribution to first author.
}

Xylanases are a major group of industrial enzymes due to their biotechnological utility and potential application in a range of industrial processes, such as biobleaching in the paper and pulp industry, bioconversion of lignocellulosic material and agro-wastes to fermentative products, clarification of juices, and improvement in the consistency of beer and the digestibility of animal feed stock (Subramaniyan and Prema, 2002). In the past few years, the potential applications of xylanases for bioconversion of lignocellulosic feedstocks to fuel-grade ethanol have been of particular interest to researchers. Current commercial preparations in lignocellulose hydrolysis have primarily been based on dilute-acid pretreatment where hemicellulose is removed before saccharification. With the development of non-acid pretreatment methods where the hemicellulose fraction remains intact, however, xylanases are required (Gray et al., 2006). Although the cost of commercial xylanase preparations has been reduced significantly in recent years, enzyme costs are still an obstacle to full-scale process commercialization (Lynd et al., 2005). Immobilization on an inert carrier offers the prospect of significant cost savings by facilitating enzyme recycling through multiple cycles of batch-wise hydrolysis (Hudson et al., 2005). Also, enzyme immobilization frequently results in improved thermal stability or resistance to shear inactivation ( $\mathrm{Tu}$ et al., 2006). The immobilized xylanases are usually packed in a column for convenient application; however, the disadvantage of such an immobilized xylanase column is that the solid property of the sub- 
strate xylan restricts passage through the column (Liu et al., 1997). In addition, the efficiency of the xylanase is decreased when immobilized on solid supports as the bound enzyme is not accessible to bulky insoluble substrate (Tan et al., 2008).

A recently established expression/purification system based on artificial oil bodies (AOBs) provides a novel method of enzyme immobilization (Peng et al., 2004; Chiang et al., 2006). In this system, oleosin, a unique structural protein of seed oil bodies, is used as the carriers. Oleosins possess a lipophilic segment embedded into the triacylglycerol core, with two amphipathic arms protruding on the surface of the oil bodies. Thus, the target protein can be expressed in Escherichia coli as an insoluble recombinant protein fused to the N- and/or C-terminus of oleosin. Stable artificial oil bodies are then constituted with triacylglycerol, phospholipid and the insoluble oleosin fusion protein. As illustrated previously, the insoluble recombinant proteins fused to oleosin tend to self-refold spontaneously when displayed on AOBs (Chiang et al., 2005). Thus, this may offer an easy and efficient, single-step method for achieving immobilization of the recombinant proteins (Chiang et al., 2006).

In the present study, a xylanase gene, $x y n C D B F V$, from the ruminal fungus Neocallimastix patriciarum was cloned and expressed as an oleosin-fused protein in E. coli. As a result of reconstitution of the AOBs, the immobilization of active xylanase was successfully accomplished. Response surface methodology (RSM) was then employed for the planned statistical optimization of the immobilized xylanase activity.

\section{Methods}

\subsection{Bacterial culture and DNA manipulation}

E. coli were grown at $37^{\circ} \mathrm{C}$ in Luria-Bertani (LB) broth (Difco Laboratories, Detroit, MI). Agar plates were prepared by adding agar $(1.5 \% \mathrm{w} / \mathrm{v})$ (Difco) to broth. Plasmid DNA was isolated from E. coli using the alkali lysis method (Birnboim and Doly, 1979). Restriction enzymes and T4 DNA ligase (New England BioLabs Inc., Beverly, MA), were used according to the manufacturer's instructions. All other DNA manipulations were performed using established procedures (Sambrook and Russell, 2001).

\subsection{Construction of xylanase expression plasmids}

The uninterrupted DNA sequence encoding xylanase XynCDBFV (Chen et al., 2001), a thermally stable and alkalophilic mutant of the catalytic domain of $N$. patriciarum xylanase XynC (GenBank accession number AF123252), was amplified by PCR from pNZJ021 (Liu et al., 2005) using the oligonucleotide forward primer, 21F ( $5^{\prime}$ GGTGGTCATATGCAAAGTTTCTGTAGTTC $3^{\prime}$ ), and the reverse primer, 21R (5' GGTGGTTGCTCTTCTGCAATCACCAATG 3'). These two primers were designed to place an NdeI site at the $5^{\prime}$ end and an SapI site at the $3^{\prime}$ end of the PCR product, respectively. The PCR fragments encoding xynCDBFV were digested with NdeI and SapI, and then ligated with NdeI-SapI-digested pOSP2 (Chiang et al., 2005) to generate pOSP2-xyn, which was then sequenced to ensure that no errors were introduced by PCR. The resultant plasmids were used to transform E. coli BL21 (DE3) (Novagene, Madison, WI) by standard techniques (Sambrook and Russell, 2001). Transformants were selected on LB agar plates containing ampicillin $(100 \mu \mathrm{g} /$ $\mathrm{mL}$ ) (Sigma Chemical Co., St. Louis, MO).

\subsection{Expression of recombinant proteins}

E. coli BL21 transformant cells were cultured in LB broth, and cell growth was then measured turbidimetrically at $600 \mathrm{~nm}$
(OD600). To produce the recombinant protein, the overnight culture was prepared and subsequently seeded into $5 \mathrm{ml}$ of LB broth. The cell cultures were maintained at $37^{\circ} \mathrm{C}$ and induced with $100 \mu \mathrm{M}$ of isopropyl-L-D-thiogalactopyranoside (IPTG; Sigma) for protein production upon reaching OD600 of 0.5 . After $4 \mathrm{~h}$ of induction, the cells were harvested by centrifugation at $5000 \mathrm{~g}$ for $20 \mathrm{~min}$ at $4{ }^{\circ} \mathrm{C}$.

\subsection{Immobilization of xylanase on AOBs}

AOBs were prepared according to the method described by Chiang et al. (2005). The cell pellet was resuspended in $1 \mathrm{~mL}$ of $0.1 \mathrm{M}$ sodium phosphate buffer ( $\mathrm{pH} 7.4$ ), sonicated for $10 \mathrm{~min}$ with an ultrasonicator (Model XL; Misonix, Farmingdale, NY), and fractioned into supernatant and pellet parts by subsequent centrifugation. Then, $15 \mathrm{mg}$ of olive oil (Sigma) and $150 \mu \mathrm{g}$ of phospholipid (Sigma) were added into the pellet fraction of the E. coli cell lysate, and the mixture was subjected to sonication. Subsequently, the reconstituted $A O B s$ were collected after centrifugation and washed in the sodium phosphate buffer solution $(0.1 \mathrm{M}, \mathrm{pH} 7.4)$. The protein production in each step was analyzed by sodium dodecyl sulfate polyacrylamide gel electrophoresis (SDS-PAGE) (Laemmli, 1970).

\subsection{Xylanase activity assays}

Xylanase activity on the AOBs was determined using oat spelt xylan (Sigma) as the substrate. Reducing sugar released from the substrate was estimated using the DNS reagent method (Konig et al., 2002). One unit of enzyme activity was defined as that releasing $1 \mu \mathrm{mol}$ of reducing sugar equivalents per minute from the substrate under the assay conditions, with specific activity expressed as IU/mg protein. Protein concentration was determined using Lowry assay (Lowry et al., 1951) against a standard curve of bovine serum albumin, fraction V (Sigma).

\subsection{Optimum $\mathrm{pH}$ and temperature of immobilized xylanase}

The response surface model, its main effects, and the interaction between the different factors, each at different levels, can be simultaneously investigated. The central composite design (CCD) was used, with two variables, at five levels and five replicates at the central point, for a total of 13 experiments. In this experimental design, $\mathrm{pH}\left(X_{1}\right)$ and temperature $\left(X_{2}\right)$ were chosen as factors, with units of xylanase activity were denoted by $Y$. According to our preliminary experimental results, the experimental index number, and scaled and real values are shown in Table 1 . The scaled values

\section{Table 1}

Process variables used in the $C C D$, showing the treatment combinations and the mean experimental responses for $\mathrm{AOB}$-immobilized xylanase

\begin{tabular}{|c|c|c|c|c|c|}
\hline \multirow[t]{2}{*}{ Treatment } & \multicolumn{2}{|c|}{$\begin{array}{l}\text { Coded setting levels } \\
\left(X_{1}=\mathrm{pH} ; X_{2}=\mathrm{T}\right)\end{array}$} & \multicolumn{2}{|c|}{$\begin{array}{l}\text { Actual levels } \\
\left(X_{1}=\mathrm{pH} ; X_{2}=\mathrm{T}\right)\end{array}$} & \multirow[t]{2}{*}{$\begin{array}{l}\text { Xylanase activity } \\
\text { (IU/mg of total protein) }\end{array}$} \\
\hline & $X_{1}$ & $X_{2}$ & $X_{1}$ & $X_{2}$ & \\
\hline 1 & -1 & -1 & 4 & 45 & 0.78 \\
\hline 2 & 0 & -1.41 & 6 & 38.8 & 1.53 \\
\hline 3 & -1.41 & 0 & 3.17 & 60 & 0.41 \\
\hline 4 & 0 & 0 & 6 & 60 & 3.75 \\
\hline 5 & 1 & 1 & 8 & 75 & 0.39 \\
\hline 6 & 0 & 0 & 6 & 60 & 3.86 \\
\hline 7 & 0 & 0 & 6 & 60 & 3.73 \\
\hline 8 & 1.41 & 0 & 8.83 & 60 & 0.35 \\
\hline 9 & 0 & 0 & 6 & 60 & 3.67 \\
\hline 10 & 1 & -1 & 8 & 45 & 0.94 \\
\hline 11 & 0 & 1.41 & 6 & 81.2 & 0.10 \\
\hline 12 & 0 & 0 & 6 & 60 & 3.82 \\
\hline 13 & -1 & 1 & 4 & 75 & 0.48 \\
\hline
\end{tabular}

Results represent the mean of three experiments. 
were defined as follows: $X_{1}=(\mathrm{pH}-6) / 2 ; X_{2}=(T-60) / 15$. Statistical Analysis System software (SAS; version 8.1; Statistical Analysis System Institute, 1998) was used for regression analysis of the experimental data. The CCD was used to allow expression of the variables as second-order polynomial models of the form:

$Y=\beta_{0}+\beta_{1} X_{1}+\beta_{2} X_{2}+\beta_{11} X_{1}^{2}+\beta_{22} X_{2}^{2}+\beta_{12} X_{1} X_{2}+\varepsilon$

where $\beta_{0}$ is constant, $\beta_{1}$ and $\beta_{2}$ are the main effect of each process variable ( $\mathrm{pH}$ and temperature), $\beta_{12}$ is the interaction effect between the variables, $\beta_{11}$ and $\beta_{22}$ are effect of the square of the variables, $Y$ is xylanase activity, $X_{1}$ and $X_{2}$ represent the independent variables ( $\mathrm{pH}$ and temperature), and $\varepsilon$ is the random error.

Finally, a series of experiment was conducted in triplicate and repeated three times in order to check the reliability of the response surface model, with the predicted values and experimental data compared. The results were analyzed using the SAS analysis of variance (ANOVA) function. Duncan's multiple range test (Montgomery, 1999) was used to detect differences between the predicted and observed values.

\subsection{Thermal stability of immobilized xylanase}

The thermal stability of AOB-immobilized xylanase was determined by incubation at $60{ }^{\circ} \mathrm{C}$ in phosphate buffer ( $\mathrm{pH}$ 6.0) containing $0.5 \%$ oat spelt xylan as the substrate. Aliquots were withdrawn at intervals of $0,20,40,60,80,100$, and $120 \mathrm{~min}$, and the residual enzyme activity measured.

\subsection{Reusability of immobilized xylanase}

The reusability of AOB-immobilized xylanase was determined by incubation at $60{ }^{\circ} \mathrm{C}$ for $20 \mathrm{~min}$ in phosphate buffer (pH6.0) containing $0.5 \%$ oat spelt xylan as the substrate. At the end of the reaction, the immobilized enzyme was recovered by centrifugation and the supernatant was removed for further analysis. The reaction was reinitiated by administration of the recovered enzymes in a fresh substrate solution.

\section{Results and discussion}

\subsection{Immobilization of xylanase XynCDBFV on AOBs}

The uninterrupted DNA fragments encoding xylanase XynCDBFV, a thermally stable and alkalophilic mutant of the catalytic domain of $N$. patriciarum xylanase XynC (GenBank accession number AF123252), were amplified by PCR and subcloned into the AOB expression/purification system vector pOSP2. XynCDBFV was overexpressed in $E$. coli as a recombinant protein fused to the $\mathrm{N}$-terminus of oleosin by a linker polypeptide. After induction with IPTG at $37^{\circ} \mathrm{C}$, the induced and non-induced recombinant bacteria containing the xylanase gene were analyzed using SDS-PAGE. A band of about $61 \mathrm{kDa}$ corresponding to the XynCDBFV-oleosin fusion protein was observed in the induced recombinant bacteria (Fig. 1, lane 2). The XynCDBFV-oleosin fusion protein was predominately found in the insoluble fraction of cell lysate after centrifugation (Fig. 1, lanes 3 and 4). After reconstitution into AOBs, the XynCDBFV-oleosin fusion protein was almost entirely present in the oil-body fraction after centrifugation (Fig. 1, lanes 5 and 6). Further examination of the reconstituted AOBs by light microscopy showed that these AOBs were extremely stable and maintained integral for at least 7 days at $4{ }^{\circ} \mathrm{C}$ (results not shown). The DNS method was then used to confirm xylanase activity of the reconstituted AOBs. A detectable level of xylanase activity was observed in the reconstituted $\mathrm{AOBs}$ from the induced recombinant bacteria

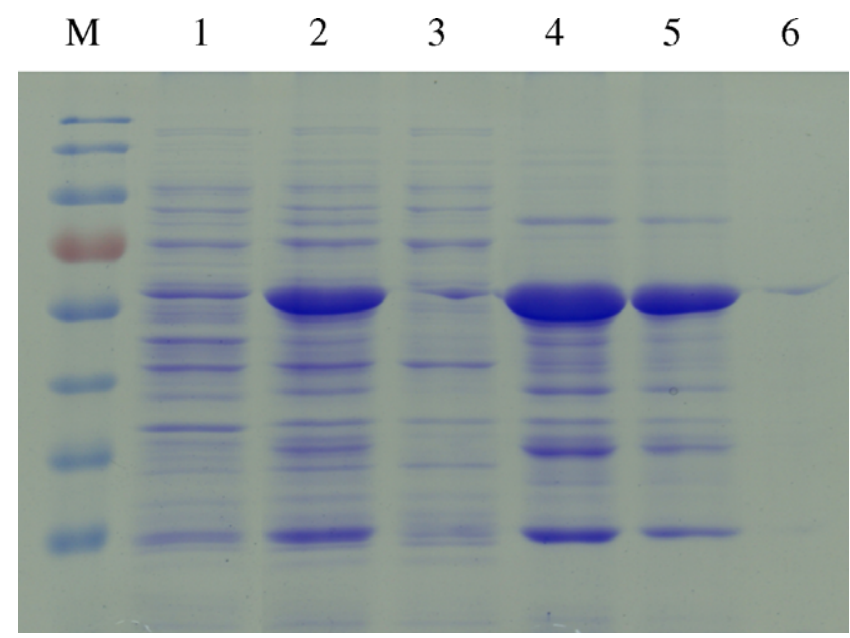

Fig. 1. SDS-PAGE analysis of XynCDBFV immobilized on AOBs. Lane M, molecular weight marker; lane 1, cell lysate of the recombinant $E$. coli before IPTG induction; lane 2, cell lysate of the recombinant $E$. coli after IPTG induction; lane 3, soluble fraction of the cell lysate after centrifugation; lane 4, insoluble fraction of the cell lysate after centrifugation; lane 5, AOBs; lane 6, remaining supernatant after AOBs recovery.

(data not shown), indicating that XynCDBFV might be immobilized on the AOB surface and fold into a correct structure. Many intrinsic soluble proteins overexpressed in E. coli are inclined to form insoluble aggregates confined in inclusion bodies. Nevertheless, the AOB system used in this study provides excellent machinery to functionally recover intrinsic soluble proteins that are overexpressed as insoluble aggregates in E. coli. Our results suggest that the folding of XynCDBFV was first tangled with the hydrophobic fusion oleosin. Constitution of AOB attracts the hydrophobic oleosin portions into the TAG matrix, thus leaving XynCDBFV alone for selfrefolding right after the physical segregation of oleosin and XynCDBFV. As XynCDBFV self-folds into its correct conformation on the surface of an $A O B$, its functional activity may be recovered concurrently.

Many reports on the immobilization of xylanase have been published (Roy et al., 2003; Hudson et al., 2005; Tan et al., 2008). The immobilized xylanases are usually packed in a column for convenient application. However, the main disadvantage for an immobilized xylanase column is that the solid property of the substrate xylan makes it hard to move through the column (Liu et al., 1997). In addition, efficiency of xylanase conversion is decreased when immobilized on solid supports as the bound enzyme is not accessible to the bulky insoluble substrate (Tan et al., 2008). Compared to conventional immobilization methods, the use of AOBs as immobilization matrices reduces the requirement for xylanase purification and eliminates the cost of immobilization. Particularly, xylanases immobilized on AOBs can be easily recovered from the surface of the solution simply by a brief centrifugation. These results prove that AOBs immobilization is a comparatively simple and effective method of direct immobilization of xylanases.

\subsection{Optimization of immobilized xylanase activity}

Base on the previous study, temperature and $\mathrm{pH}$ were identified as the major factors affecting the activity of xylanase XynCDBFV (Chen et al., 2001). RSM is an empirical modeling technique used to evaluate the relationship between a set of controllable experimental factors and observed results. The CCD experimental design, which minimizes the number of experimental runs, was used to determine the effects of independent variables on the dependent variables. The process variables used in the experimental design 
and results for enzyme activities are shown in Table 1 . Treatments $4,6,7,9$ and 12 (central points) showed the highest levels of xylanase activity $(3.75,3.86,3.73,3.67$, and $3.82 \mathrm{IU} / \mathrm{mg}$ of total protein, respectively). These results suggest that the immobilized xylanase has greater enzyme activity at higher temperatures and moderate acidic $\mathrm{pH}$.

Analysis of variance (ANOVA) of immobilized xylanase activity as a function of $\mathrm{pH}$ and temperature is presented in Table 2. The computed $F$-value (456.57) was higher than the analogous value in the statistical tables $(<0.0001)$, indicating that the model was highly significant (Box and Wilson, 1951). The goodness of fit of the model was checked using the coefficient of determination $\left(R^{2}=0.9969\right)$, indicating that just $0.31 \%$ of the total variation was not explained by the model. This confirms that the accuracy and general ability of the quadratic model was good, with the analysis of the associated response trends considered to be reasonable. In addition, the lack-of-fit test was insignificant, suggesting that the model accurately represents the data in the experimental region.

RSM yielded the following regression equation:

$$
\begin{aligned}
Y= & -32.56+5.34^{*} \mathrm{pH}+0.69^{*} T-0.43^{*} \mathrm{pH}^{2}-0.005761^{*} T^{2} \\
& -0.002125^{*} \mathrm{pH}^{*} T
\end{aligned}
$$

where $Y$ is the predicted response for xylanase activity (IU/mg of total protein), and $\mathrm{pH}$ and $T$ are the coded values for $\mathrm{pH}$ and temperature (see Table 1 ).

The significance of the coefficients determined by Student's $t$ test and the related $P$-values are presented in Table 3 . The latter were used to check the significance of each coefficient, and also indicated the strength of the interaction between each independent variable (i.e., the smaller the $P$-value, the more significant the corresponding coefficient) (Akhnazarova and Kafarov, 1982). In this study, the $P$-value of $\mathrm{pH}$, second-order $T$, and the interaction coefficient of $\mathrm{pH}$ and $T$ were highly significant $(P<0.005)$. The high significance of the $T$ second-order model indicates that it can act as a limiting factor, with even small variations substantially altering xylanase activity (Heck et al., 2006). The model also clearly reveals significant interactions between $\mathrm{pH}$ and $T(P<0.0001)$, therefore, treating them separately may not reflect their real influence on the xylanase activity (e.g., optimum $T$ activity changes along with $\mathrm{pH})$.

Table 2

Analysis of variance (ANOVA) for model regression

\begin{tabular}{llllll}
\hline $\begin{array}{l}\text { Source of } \\
\text { variation }\end{array}$ & $\begin{array}{l}\text { Sum of } \\
\text { squares }\end{array}$ & $\begin{array}{l}\text { Degree of } \\
\text { freedom }\end{array}$ & $\begin{array}{l}\text { Mean } \\
\text { square }\end{array}$ & $\begin{array}{l}F- \\
\text { value }\end{array}$ & $\begin{array}{l}F \text {-value in } \\
\text { statistic table }\end{array}$ \\
\hline Model & 29.410 & 5 & 5.880 & 456.57 & $<0.0001$ \\
Residual & 0.090 & 7 & 0.013 & & \\
Lack of fit & 0.067 & 3 & 0.022 & 3.96 & 0.1086 \\
Pure error & 0.023 & 4 & 0.00569 & & \\
Total & 29.50 & 12 & & &
\end{tabular}

Model regression is $Y=-32.56+5.34^{*} \mathrm{pH}+0.69^{*} T-0.43^{*} \mathrm{pH}^{2}-0.005761^{*} T^{2}-$ $0.002125^{*} \mathrm{pH}^{*} T$.

$R^{2}=0.9969$; standard error of estimate $=0.0005$; significance level $=95 \%$.

Table 3

Coefficient estimates by the regression model

\begin{tabular}{llll}
\hline Factor & Coefficient estimate & Standard error & $P$-value \\
\hline Intercept & 3.770 & 0.051 & \\
$T$ & -0.002 & 0.040 & 0.9674 \\
$\mathrm{pH}^{*}$ & -0.200 & 0.040 & 0.0016 \\
$T \cdot T^{*}$ & -1.740 & 0.043 & $<0.0001$ \\
$T \cdot \mathrm{pH}^{*}$ & -1.300 & 0.043 & $<0.0001$ \\
$\mathrm{pH} \cdot \mathrm{pH}$ & -0.064 & 0.057 & 0.2984 \\
\hline
\end{tabular}

Statistically significant at $99 \%$ of confidence level.
The RSM for enzyme activity as a function of $\mathrm{pH}$ and temperature of the immobilized xylanase is depicted in Fig. 2. The results indicate that the optimal conditions for the immobilized xylanase activity occur at $59{ }^{\circ} \mathrm{C}$ and $\mathrm{pH}$ 6.0. To confirm the applicability of the model, xylanase activity at the suggested optimum condition was determined. In this condition, the model predicted enzymatic activity of $3.93 \mathrm{IU} / \mathrm{mg}$ at a confidence level of 95\%. The experimental enzymatic activity of $3.90 \mathrm{IU} / \mathrm{mg}$ confirmed the accuracy of the model.

The general utility of xylanases in industry has spurred considerable research into the production of more thermally stable and alkalophilic variants. The xylanase, XynCDBFV, used in this study is a thermally stable and alkalophilic mutant of the catalytic domain of $N$. patriciarum xylanase XynC. It was previously determined that the optimum temperature and $\mathrm{pH}$ of free XynCDBFV was $62{ }^{\circ} \mathrm{C}$ and $\mathrm{pH} 6.0$, respectively (Chen et al., 2001). In this study, the maximum activity of immobilized XynCDBFV was determined at $59{ }^{\circ} \mathrm{C}$ and $\mathrm{pH}$ 6.0. Therefore, immobilization of the XynCDBFV on AOBs did not significantly change the optimum $\mathrm{pH}$ of the xylanase activity, and only slightly decreased the optimum temperature. At $\mathrm{pH} 8.5,47^{\circ} \mathrm{C}$, and at $\mathrm{pH} 6.0,77^{\circ} \mathrm{C}$, the free XynCDBFV maintained approximately $45 \%$ and $10 \%$ of maximal activity, respectively (Chen et al., 2001), while the analogous maxima for the immobilized form were $16 \%$ and $51 \%$ in this study. Thus, it appear reasonable to suggested that immobilization on AOBs may improve the thermal stability of XynCDBFV but not its alkalinity tolerance.

\subsection{Thermal stability of immobilized xylanase}

Thermal stability is very important in the industrial application of enzymes. In terms of the thermal stability of the immobilized XynCDBFV in our study, $50 \%$ of maximal activity was retained after $120 \mathrm{~min}$ at $60^{\circ} \mathrm{C}$. However, this result contrasts with that of Chen et al. (2001), who found that the activity of free XynCDBFV was reduced to $30 \%$ of maximum at the same time and temperature. Thus, better thermal stability was demonstrated for the immobilized XynCDBFV in our study which compared to the free enzyme. Chiang et al. (2006) immobilized Agrobacterium radiobacter D-hydantoinase on AOBs and also demonstrated that the immobilized enzyme exhibited a higher thermal tolerance than the free enzyme. In addition, Li et al. (2007) immobilized cellulase on the outer membrane of liposomes and indicated that the liposome membrane was favorable for maintaining the activity of the cellulase in these cellulase-containing liposomes. It appears reasonable to suggest, therefore, that the interaction between enzyme and oil-

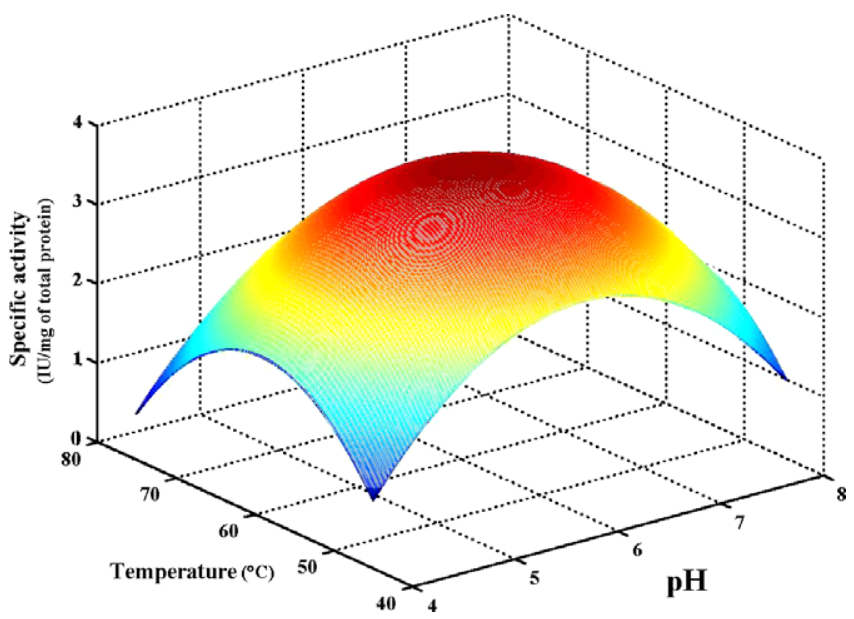

Fig. 2. Response surface plot for the effects of $\mathrm{pH}$ and temperature on AOB-immobilized xylanase activity. 
body membrane is beneficial in terms of reducing the enzyme deactivation.

\subsection{Reusability of immobilized xylanase}

Immobilized enzymes are preferred as in this form they can be recycled, thereby reducing production costs. Further, AOBs can be separated from the reaction mix by flotation centrifugation (Chiang et al., 2006), facilitating the recovery and recycling of the xylanase immobilized thereon. The recovery and operational stability of AOB-immobilized XynCDBFV was examined during eight successive rounds of xylan hydrolysis. The AOB-immobilized XynCDBFV could be reused for three cycles at $60^{\circ} \mathrm{C}$ without loss in activity. After that, the enzyme activity gradually declined and still retained more than $60 \%$ of initial activity after eight-time recycling of the enzyme (data not shown). It is interesting to note that because xylanase immobilized on AOBs floats rather than sinks, it may be much easier to recover in heterogeneous mixtures which contain precipitable solids, which could confound attempts to recover enzymes bound to solid matrices. However, evaluation of the operational stability of enzymes is necessary as this property is one of the most important factors affecting the successful applications of immobilized systems. Liu et al. (1997) fused the $N$. patriciarum xylanase gene xynC to an oleosin gene, and then introduced the fusion gene into Brassica napus. These workers found that XynC was immobilized on the surface of oil-bodies extracted from the transgenic canola seeds, and that it could be reused several times without impairment of the xylanase activity (Liu et al., 1997). In this study, XynCDBFV was immobilized on AOBs, and it was demonstrated that AOB-immobilized XynCDBFV could be reused eight times and still retain more than $60 \%$ of its activity. It appears reasonable to suggest, therefore, that these results prove that xylanase immobilized on AOBs can be reused, thereby reducing the cost of enzymes of industrial processes where the cost of enzyme is significant.

\section{Conclusions}

The ruminal fungus $N$. patriciarum xylanase gene, xynCDBFV, was cloned and expressed as an oleosin-fused protein in E. coli. Immobilization of active xylanase was accomplished as a result of reconstituting AOBs, with RSM employed for the planned statistical optimization of the immobilized xylanase activity. It was demonstrated that $C C D$ and regression analysis methods were effective in determining optimized temperature and $\mathrm{pH}$ conditions for AOB-immobilized XynCDBFV. In this study, the optimal conditions for the immobilized xylanase activity were $59{ }^{\circ} \mathrm{C}$ and $\mathrm{pH}$ 6.0. In addition, it was demonstrated that AOB-immobilized XynCDBFV can be easily and simply recovered from the surface of the solution by brief centrifugation, and that it can be reused eight times while retaining more than $60 \%$ of its activity. These results prove that immobilization of xylanase on AOBs is a comparatively straightforward and effective means of direct immobilization of xylanases.

\section{Acknowledgements}

This research was conducted using funds partially provided by Grant NSC 95-2313-B-002-118-MY3 and NSC 96-2622-B-002-
006-CC3 from the National Science Council and Grant 96AS2.1.3-AD-U1(7) from the Council of Agriculture, Republic of China.

\section{References}

Akhnazarova, S., Kafarov, V., 1982. Experiment Optimization in Chemistry and Chemical Engineering. Mir, Moscow.

Birnboim, H.C., Doly, J., 1979. A rapid alkaline extraction procedure for screening recombinant plasmid DNA. Nucleic Acids Res. 7, 1513-1517.

Box, G.E.P., Wilson, K.B., 1951. On the experimental attainment of optimum conditions. J. R. Stat. Soc. 13, 1-45.

Chen, Y.L., Tang, T.Y., Cheng, K.-J., 2001. Directed evolution to produce an alkalophilic variant from a Neocallimastix patriciarum xylanase. Can. J. Microbiol. 47, 1088-1094.

Chiang, C.J., Chen, H.C., Chao, Y.P., Tzen, J.T.C., 2005. Efficient system of artificial oil bodies for functional expression and purification of recombinant nattokinase in Escherichia coli. J. Agric. Food Chem. 53, 4799-4804.

Chiang, C.J., Chen, H.C., Kuo, H.F., Chao, Y.P., Tzen, J.T.C., 2006. A simple and effective method to prepare immobilized enzymes using artificial oil bodies. Enzyme Microb. Technol. 39, 1152-1158.

Collins, T., Gerday, C., Feller, G., 2005. Xylanases, xylanase families and extremophilic xylanases. FEMS Microbiol. Rev. 29, 3-23.

Gray, K.A., Zhao, L., Emptage, M., 2006. Bioethanol. Curr. Opin. Chem. Biol. 10, 141146.

Heck, J.X., Flôres, S.H., Hertz, P.F., Ayub, M.A.Z., 2006. Statistical optimization of thermo-tolerant xylanase activity from Amazon isolated Bacillus circulans on solid-state cultivation. Bioresour. Technol. 97, 1902-1906.

Hudson, S., Magner, E., Cooney, J., Hodnett, B.K., 2005. Methodology for the immobilization of enzymes onto mesoporous materials. J. Phys. Chem. B 109 19496-19506.

Konig, J., Grasser, R., Pikor, H., Vogel, K., 2002. Determination of xylanase, $\beta$ glucanase, and cellulase activity. Anal. Bioanal. Chem. 374, 80-87.

Kulkarni, N., Shendye, A., Rao, M., 1999. Molecular and biotechnological aspects of xylanases. FEMS Microbiol. Rev. 23, 411-456.

Laemmli, U.K., 1970. Cleavage of structural proteins during the assembly of the head of bacteriophate T4. Nature 227, 680-685.

Li, C., Yoshimoto, M., Fukunaga, K., Nakao, K., 2007. Characterization and immobilization of liposome-bound cellulase for hydrolysis of insoluble cellulose. Bioresour. Technol. 98, 1366-1372.

Liu, J.H., Selinger, L.B., Cheng, K.-J., Beauchemin, K.A., Moloney, M.M., 1997. Plant seed oil-bodies as an immobilization matrix for a recombinant xylanase from the rumen fungus Neocallimastix patriciarum. Mol. Breeding 3, 463-470.

Liu, J.R., Yu, B., Liu, F.H., Cheng, K.-J., Zhao, X., 2005. Expression of rumen microbial fibrolytic enzyme genes in probiotic Lactobacillus reuteri. Appl. Environ. Microbiol. 71, 6769-6775.

Lowry, O.H., Rosebrough, N.J., Farr, A.L., Randall, R.J., 1951. Protein measurement with the folin-phenol reagent. J. Biol. Chem. 193, 256-275.

Lynd, L.R., van Zyl, W.H., McBride, J.E., Laser, M., 2005. Consolidated bioprocessing of cellulosic biomass: an update. Curr. Opin. Biotechnol. 16, 577-583.

Montgomery, D.C., 1999. Experiments with a single factor: the analysis of variance. In: Montgomery, D.C. (Ed.), Design and Analysis of Experiments. John Wiley and Sons, New York, pp. 75-77.

Peng, C.C., Shyu, D.J.H., Chou, W.M., Chen, M.J., Tzen, J.T.C., 2004. Method for bacterial expression and purification of sesame cystatin via artificial oil bodies. J. Agric. Food Chem. 52, 3115-3119.

Roy, I., Gupta, A., Khare, S.K., Bisaria, V.S., Gupta, M.N., 2003. Immobilization of xylan-degrading enzymes from Melanocarpus albomyces IIS 68 on the smart polymer Eudragit L-100. Appl. Microbiol. Biotechnol. 61, 309-313.

Sambrook, J., Russell, D.W., 2001. Molecular Cloning: A Laboratory Manual, third ed. Cold Spring Harbor Laboratory Press, Cold Spring Harbor, NY.

Statistical Analysis System Institute, 1998. SAS User's Guide: Statistics. SAS Institute Inc., Cary, N.C.

Subramaniyan, S., Prema, P., 2002. Biotechnology of microbial xylanases: enzymology, molecular biology, and application. Crit. Rev. Biotechnol. 22, 3364.

Tan, S.S., Li, D.Y., Jiang, Z.Q., Zhu, Y.P., Shi, B., Li, L.T., 2008. Production of xylobiose from the autohydrolysis explosion liquor of corncob using Thermotoga maritima xylanase B (XynB) immobilized on nickel-chelated Eupergit C. Bioresour. Technol. 99, 200-204.

Tu, M., Zhang, X., Kurabi, A., Gilkes, N., Mabee, W., Saddler, J., 2006. Immobilization of $\beta$-glucosidase on Eupergit C for lignocellulose hydrolysis. Biotechnol. Lett. 28, $151-156$.

Wu, S., Liu, B., Zhang, X., 2006. Characterization of a recombinant thermostable xylanase from deep-sea thermophilic Geobacillus sp. MT-1 in East Pacific. Appl. Microbiol. Biotechnol. 72, 1210-1216. 